\title{
LITHIUM NIOBATE-BASED INTEGRATED PHOTONICS UTILIZING PHOTOREFRACTIVE EFFECT
}

\begin{abstract}
Lithium niobate $\left(\mathrm{LiNbO}_{3}\right)$ is an ideal material with many interesting properties for integrated photonics. Despite the rich set of properties the technology of lithium niobate integrated optics has not evolved as much as integrated optics in III-V semiconductors and silicon photonics. Future applications of $\mathrm{LiNbO}_{3}$ as an integrated optical platform require a technology that can materialize ultracompact and efficient optical circuits on the material. To achieve the goal two possible approaches can be considered: developing of tightly-confined lithium niobate photonic devices and circuits on silicon substrates by hybrid technologies and, developing pure lithium niobate photonic devices employing strong photorefractive effect. The latter approach is in more details discussed in the contribution and concrete examples of practically realized photonic structures are presented.
\end{abstract}

Keywords: Lithium niobate, integrated optics, photonic integrated circuit, photorefractive effect, waveguide.

\section{Introduction}

The development of the laser in the 1960s opened the possibility to transmit and process signals carried by optical beams. Since then the idea has attracted many researchers. The primary problems regarding a proper medium for signal transmission and optical components for signal processing were solved rather soon. As a result, the concept of integrated optics emerged, in which the through-the-air optical paths commonly used in laboratories were replaced by dielectric light-guides and conventional electrical integrated circuits were replaced by optical integrated circuits (OIC's) or photonic integrated circuits (PIC's). A decade later the development of low-loss optical fibers and connectors, creation of semiconductor laser diodes (e.g. GaAlAs and GaInAsP) and huge advances in photolithographic microfabrication techniques as well, helped to bring the integrated optics into real life and solve many practical problems. Moreover, in last two decades the microtechnology naturally evolved into nanotechnology giving birth to nanophotonics the important part of which is the fabrication of photonic crystals [1].

The OIC has a number of advantages compared to conventional electrical integrated circuit [2]: increased bandwidth; expanded frequency (wavelength) division multiplexing; lowloss couplers; expanded multipole switching; smaller size; light weight; low power consumption; batch fabrication economy; improved reliability; improved optical alignment; immunity from electromagnetic interference; immunity to vibration; safety in combustible environment; freedom from electrical short circuits or ground loop, etc. The major disadvantages of OIC's can be considered difficulty to use for electrical power transmission and high cost of developing new fabrication technology.

\subsection{Material basis for OIC's and fabrication approach}

The choice in which material fabricate an optical integrated circuit depends mostly on the function to be performed by the circuit. The OIC may be required to consist of different optical devices, passive and/or active. However, none of the material is suitable for both kinds of the devices working at once so a compromise must be made. This is reflected also in the OIC's fabrication.

There are two basic approaches to fabricate an OIC. One of these is the monolithic in which a single material is used for all devices. Monolithic circuits requiring a source of light can only be fabricated in active materials, mainly semiconductors such as GaAs, GaAlAs, GaAsP, GaInAs and other III-V and II-VI semiconductors. Passive materials like quartz, lithium niobate or polymers are also useful as substrate materials but generally an external light source, such as a semiconductor laser, must somehow be optically and mechanically coupled to the substrate [2].

The other approach is a hybrid one in which two, but usually more, materials are technologically bonded together

\footnotetext{
* Norbert Tarjanyi, Daniel Kacik

Department of Physics, Faculty of Electrical Engineering, University of Zilina, Slovakia

E-mail: tarjanyi@fyzika.uniza.sk
} 
and optimized for performance of different integrated devices. The major advantage of the approach is that the OIC's can be fabricated using existing technology, piecing together devices which have been substantially optimized in a given material. The main disadvantage is that the bonds between the various elements of the circuit are subject to misalignment, or even failure, because of vibration and thermal expansion. Also, the monolithic approach is ultimately cheaper if mass production of the circuit is desired, because of the usage of automated batch processing.

\section{Lithium niobate as a basis for integrated photonics}

Lithium niobate is an ideal material with many interesting properties for integrated photonics. First, it is transparent from the UV to IR range of the electromagnetic spectrum. Doping the crystal with rare earth elements, such as erbium can lead to optical gain. A unique advantage is the material's strong secondorder nonlinear optical properties, which allows the control of the refractive index of the material via the electro-optic effect. The nonlinearity also allows mixing of optical signals at different wavelengths for parametric amplification, second-harmonic generation [3], wavelength conversion, as well as generation of entangled photon pairs [4]. Using the piezo-electric properties of $\mathrm{LiNbO}_{3}$, it is possible to make various acousto-optical devices but nowadays, the most widely used devices based on $\mathrm{LiNbO}_{3}$ are high-speed electro-optical modulators [5, 6].

As optical components continue to replace its electrical counterparts in various signal processing applications there is a growing demand to integrate more photonic devices onto a single chip. In the last few years strong efforts have been made to develop silicon based photonic chips [7]. However, the physical limitations of the material and demanding technology as well, naturally led to an idea combine the advantages of various traditional materials and other technologies also used in photonics. The result is a hybrid technology called "lithium niobate on insulator" (LNOI) which is very promising for preparation of photonic circuits and their components [7 - 11]. The monolithic fabrication technology is still mainly used for preparation of passive PIC's on $\mathrm{LiNbO}_{3}$ substrate. Here, several quite different approaches can be distinguished like [e.g. 12]: local doping followed by thermal diffusion, proton exchange, ion implantation; ferroelectric domain engineering; use of lightinduced phenomena including solitons [12]. Nowadays, first three approaches represent a well-established techniques and a number of various kinds of waveguides and wave guiding structures was prepared using them up to date. The last two approaches are rather new and offer new possibilities hardly achievable by traditional techniques. Recently, the last approach mentioned is of a great interest as, in principle, it does not require any complicated supplementary technology, and is chemicals free. As a laser light with the proper wavelength, power and gradient of intensity is used to induce processes within the $\mathrm{LiNbO}_{3}$ crystal leading to required local change of its optical properties the technique is often regarded as direct laser writing (DLW) [13 - 16].

\section{DLW technique of fabrication PIC's and their components}

In lithium niobate the direct laser writing technique can be performed in visible using a proper continuous wave laser light source as well as in ultraviolet or infrared regions of electromagnetic spectrum, using ultra-short laser pulses. Even if there are numerous analogies such as the setup scheme, the dependence on the crystal orientation and the obtainable structures, the mechanisms responsible mainly for refractive index change are different. In the case the changes are induced due to visible light the effect is called photorefractive. Actually, the photorefractive effect is a combination of several mechanisms including optical ionization of certain impurities, spatial redistribution of released charge carriers which gives rise to internal electric field and finally, linear electro-optic effect [17]. Further, we will limit our discussion to photorefractive effect only as it is still the most common phenomenon used for fabrication of photonic structures. Recently, we have investigated the effect in various $\mathrm{LiNbO}_{3}$ crystals and, besides the investigation, also created some applications useful for the implementation into photonic integrated circuits. An overview that follows documents exceptional technological potential of the photorefractive phenomenon.

\subsection{Photorefractive gratings}

The most common structure produced in a $\mathrm{LiNbO}_{3}$ crystal is a holographic grating created due to illumination of the crystal by light with harmonic spatial distribution of intensity. The desired distribution of intensity of light can be simply obtained by interference of two laser beams (we usually used the blue line of $\mathrm{Ar}$ ion laser) [1]. Since the setup is similar to that used in holography when recording a hologram, the produced grating is often called the holographic one. The existence of the recorded grating can be proved by diffraction of an extra light beam of a different wavelength (e.g. red line of $\mathrm{He}-\mathrm{Ne}$ laser) as was the wavelength of the recording beams or by putting the crystal into one arm of Mach-Zehnder interferometer, for example, or both [18]. As the grating is represented by a region of spatially modulated refractive index, it behaves like a phase object and can be imaged by means of an interferometer. For illustration, the spatial distribution of the refractive index change representing a grating imaged in this way is shown in Fig. 1a.

In principle, the grating can work in either transmission (Fig. 1b) or reflection (Fig. 1c) regimes depending on desired 


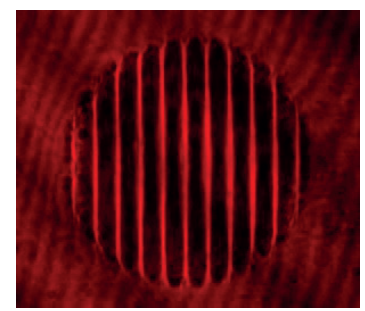

(a)

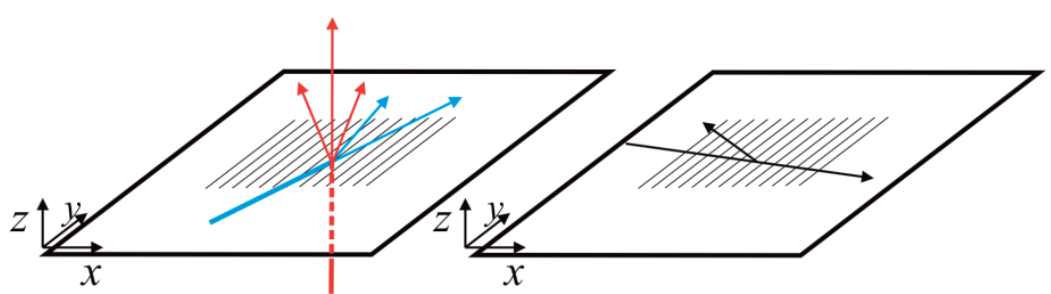

(b)

(c)

Fig. 1 The refractive index distribution representing the photorefractive grating with a spatial period $\Lambda=200 \mu \mathrm{m}$ recorded in $1 \mathrm{~mm}$ thick sample of $\mathrm{LiNbO}_{3}$ imaged by Mach-Zehnder interferometer (a). Photorefractive grating can be used to work in the transmission regime (b) or the reflection regime $(c)$.

function of the device. In the transmission regime, the wavelength dependence of diffraction of light on the grating with constant spatial period leads simply to spatial separation of signals with different wavelengths, which can be used for demultiplexing and redirecting of those signals. However, in a real life the situation might become a bit complicated in case the grating is thick (one cannot neglect the thickness of the grating with respect to grating spacing) because of energy coupling between diffraction orders. In the reflection regime the same grating will show the wavelength selectivity, which can be used for signal filtering.

Depending on the wavelength of the recording beams and the mutual angle between beams we can conveniently create gratings with various spatial periods in the range from few micrometers up to few hundreds of micrometers. Another important parameter of a grating is its diffraction efficiency. For reflection grating the diffraction efficiency is function of grating spacing, amplitude of refractive index modulation $\Delta n$ and wavelength of light. As an example the diffraction efficiencies (the so called reflection spectra) for the reflection grating with grating spacing of $1 \mathrm{~mm}$ and length $10 \mathrm{~mm}$ are shown in the Fig. 2.

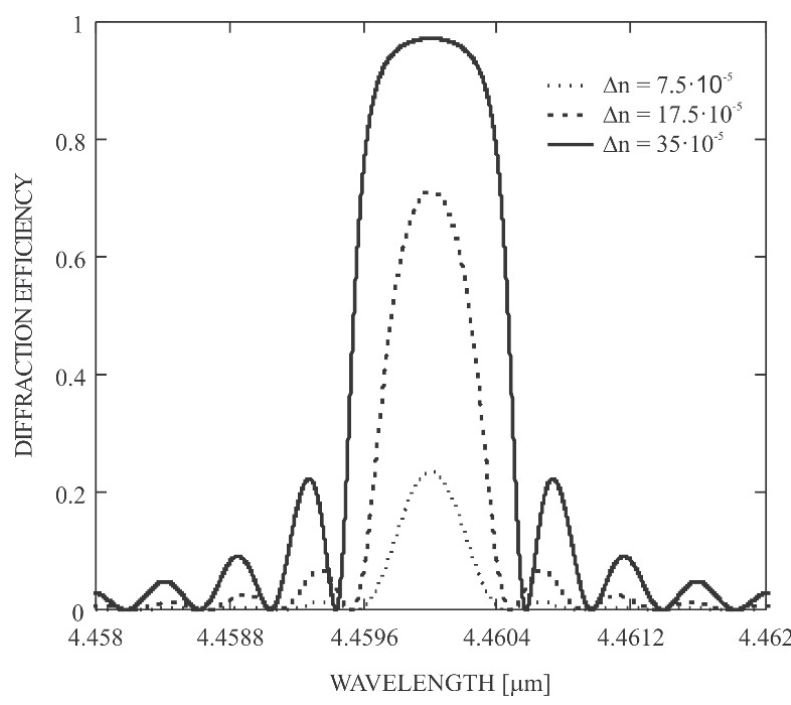

Fig. 2 Diffraction efficiencies of $10 \mathrm{~mm}$ long reflection grating with grating period $\Lambda=1 \mu \mathrm{m}$ for various amplitudes of refractive index modulation $\Delta n$
The diffraction efficiencies $\eta$ were calculated according to coupled mode theory approach [19]

$$
\eta=\frac{|\kappa|^{2} \cdot \sinh ^{2}(s \cdot L)}{s^{2} \cdot \cosh ^{2}(s \cdot L)+\left(\frac{\Delta \beta}{2}\right)^{2} \cdot \sinh ^{2}(s \cdot L)},
$$

where parameters $\kappa, \Delta \beta$ and $s$ are

$\kappa=\frac{2 \pi \cdot \Delta n \cdot n_{0} \cdot \Lambda}{\lambda^{2}}$

$\Delta \beta=\frac{2 \pi}{\Lambda}-\frac{4 \pi}{\lambda_{B}} \cdot n_{o} \cdot \sin \theta_{B}=0$,

$s^{2}=|\kappa|^{2}-\left(\frac{\Delta \beta}{2}\right)^{2}$.

In Equations (1) - (4) $L$ is the length of the grating, $\Delta n$ is the refractive index modulation, $n_{0}$ is the refractive index of the crystal, $\Lambda$ is the grating period, $\lambda$ is the wavelength of light, $\lambda_{B}$ and $\theta_{B}$ are the Bragg wavelength and the Bragg angle, respectively.

In photorefractive $\mathrm{LiNbO}_{3}$ crystals the amplitude of the refractive index modulation depends on the exposure (product of intensity of recording light and time) and can reach, in the used samples, the values up to $7.5 \cdot 10^{-4}$. It can be seen from the Fig. 2 that reflection grating with $1 \mathrm{~mm}$ spacing and $10 \mathrm{~mm}$ length is set to work with high efficiency at wavelength $\lambda=4.46 \mu \mathrm{m}$. Such grating would be suitable for the devices and applications designed for mid-infrared region of electromagnetic spectrum.To get closer to NIR or even VIS region one has to set the grating spacing to the sub-micrometer values. This can be done, for example, by changing the recording geometry.

When we let the two coherent optical waves counter-propagate in the crystal, they will interfere with each other and form a standing optical wave with the spacing between two maxima being half of the wavelength of the original waves in the medium [20]. If the waves are propagating along $c$-direction of the $\mathrm{LiNbO}_{3}$ crystal, the gradient of the interference pattern will be along this direction, too. This is the welcome situation for the standing wave being successfully recorded. The counter-propagating waves can be formed for example, using one beam propagating through the crystal and reflecting on the backside of the crystal due to Fresnel reflection. The efficiency of the reflection can be increased, for 
example, by metalizing the backside of the crystal and so the modulation of the interference pattern of the standing wave can be increased, too. The calculated diffraction efficiency for such grating written by light with wavelength $\lambda=488 \mathrm{~nm}$ is shown in Fig. 3.

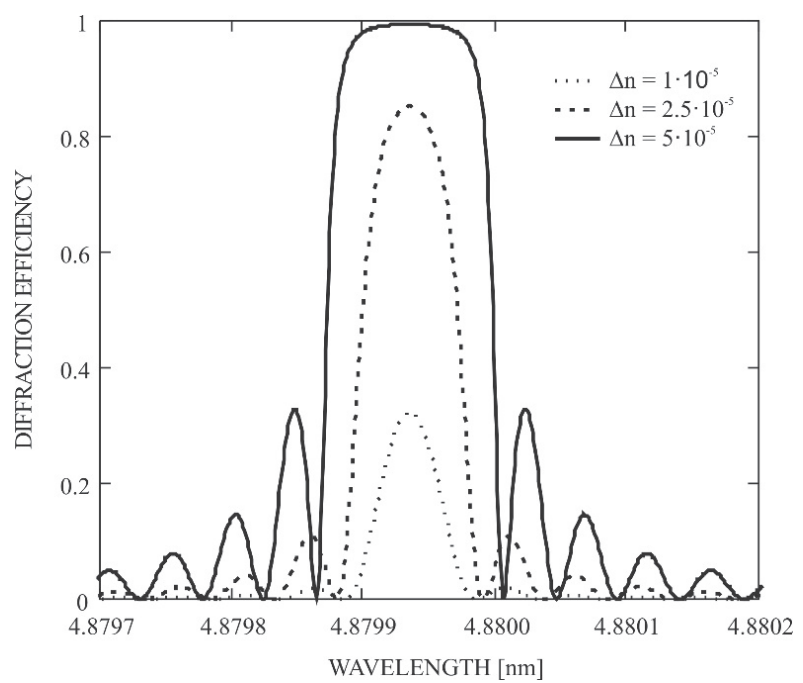

Fig. 3 Diffraction efficiency of the grating formed by standing optical wave calculated for various amplitudes of refractive index modulation $\Delta n$. Grating spacing $\Lambda \sim 0.1 \mu \mathrm{m}$, interaction length $L=10 \mathrm{~mm}$.

The disadvantage of this 'standing wave' geometry is evident: only reflection grating for wavelength to which the crystal is photosensitive can be produced. To overcome the disadvantage the gratings with sub-micrometer periods can be fabricated by point-by-point direct laser writing method which combines the stationary, focused laser beam with computer-controlled motion of the crystal or vice versa. The result is a photonic structure with desired parameters.

There is one more feature of the light induced refractive index inhomogeneity within the crystal - it also possesses the dioptric properties [21]. After illumination of the grating shown in Fig. 1a by light the phase of the light-wave behind the structure will be modified resulting in a focusing of rays into lines. The grating thus behaves like an array of cylindrical microlenses [22]. Combining two sets of such arrays oriented perpendicularly to each other will produce a microarray of spherical lenses. The spatial period of the array and the focal length of the lenses can be well controlled by the angle of interfering beams creating the grating and exposure, respectively.

\subsection{Photorefractive waveguide}

The optical waveguide is the fundamental element for the integrated photonics. Utilizing the photorefractive effect a properly structured optical field can create a waveguide in
$\mathrm{LiNbO}_{3}$ crystal. It is practical to use the optical fields with spatial symmetry being somehow related to the symmetry of the waveguide. Thus a Gaussian laser beam expanded by a cylindrical lens or such a beam spatially restricted in order to form a strip or set of strips can be considered the appropriate optical field. For the structure to behave as a waveguide must be assured that the refractive index of the guiding layer is higher than that of the surroundings. As already mentioned the best contrast in the refractive index change is between the illuminated and dark regions and is achieved when the gradient of the illumination is parallel with $c$-axis of the crystal. When the crystal is irradiated by the optical field with the Gaussian spatial distribution of the intensity modified by a cylindrical lens the inhomogeneity of the refractive index of the shape as shown in Fig. 4 is induced in the crystal. The spatial distribution of the refractive index was calculated according to the assumptions that: (i) the refractive index changes are dominantly due to electro-optic effect, (ii) the diffusion of the charge carriers is negligibly small and the photogalvanic current is dominantly contributing to the distribution of the charge density, (iii) the crystal is surrounded by an electrically conductive medium [23].

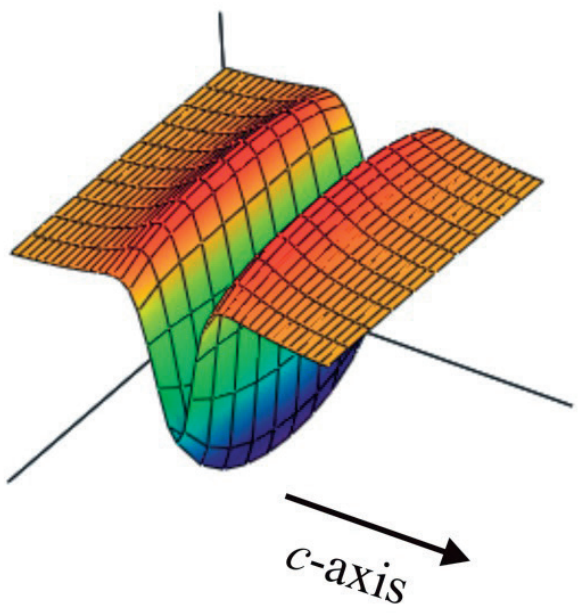

Fig. 4 Calculated refractive index change induced by an expanded Gaussian beam

The pattern shown in Fig. 4 consists in a negative refractive index variation at the core of the beam and in a two-sided lobe structure disposed along the $c$-direction with a positive refractive index change. Thus each one of these lobes with positive refractive index can be used to guide light.

We used a different approach and recorded the waveguide by means of a spatially restricted Gaussian beam modified by a cylindrical lens in two steps. Firstly, we applied the recording optical field with intensity $1.2 \mathrm{mWmm}^{-2}$ onto the crystal and set the proper recording time to achieve the steady-state value of refractive index change ( $t=300 \mathrm{~s}$ in this case). After that the sample was moved mechanically along $c$-axis of the crystal by 
about $150 \mu \mathrm{m}$ and the second exposure at the same conditions finished the process of the waveguide formation (Fig. 5) and the guiding properties were demonstrated [23, 24].

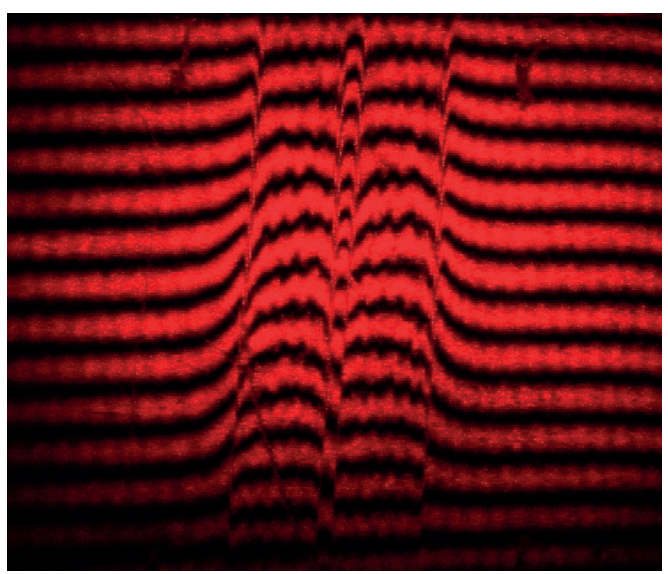

Fig. 5 Spatial distribution of refractive index in the sample with recorded waveguide imaged by means of Mach-Zehnder interferometer.

The width of the illumination strip (two wider strips in the figure) is $1 \mathrm{~mm}$. The width of the waveguiding area in between the strips is $150 \mu \mathrm{m}$

\section{Conclusion}

We presented an overview of the issue of integrated optics and photonics in general and with focus on the current state of photonics employing lithium niobate as a basis for various passive and active photonic devices. Our recent work devoted to investigation of photorefractive effect in lithium niobate showed applicability of the effect in the fabrication of passive photonic devices based on waveguides, gratings and microlens arrays. These can be combined and naturally integrated on a single lithium niobate substrate material forming a photonic integrated circuit performing e.g. waveguiding, filtering and/ or demultiplexing functions. The direct laser writing method of fabrication of the photonic devices in $\mathrm{LiNbO}_{3}$ has a big potential due to its simplicity, no need of post-processing and 'green' (chemicals free) approach.

\section{Acknowledgements}

This work was supported by Slovak National Grant Agency No. VEGA 1/0491/14, 1/0278/15 and Slovak Research and Development Agency under the project No. APVV-15-0441.

\section{References}

[1] KACIK, D., et al.: Photonic Crystals: Optical Structures for Advanced Technology. Communications - Scientific Letters of the University of Zilina, 10(2), 25-29, 2008.

[2] HUNSPERGER, R. G.: Integrated Optics: Theory and Technology, Chapter 1. Springer, New York, p. 1-12, 2009.

[3] GEISS, R., et al.: Fabrication of Nanoscale Lithium Niobate Waveguides for Second-Harmonic Generation. Optics Letters, 40(12), 2715-2718, 2015.

[4] JIN, H., et al.: On-Chip Generation and Manipulation of Entangled Photons Based on Reconfigurable Lithium-Niobate Waveguide Circuits. Physical Review Letters, 113(10), 103601-1-103601-5, 2014.

[5] WOOTEN, E.L., et al.: A Review of Lithium Niobate Modulators for Fiber-Optic Communications Systems. IEEE Journal of Selected Topics in Quantum Electronics, 6(1), 69-81, 2000.

[6] SAKAMOTO, T., et al.: Novel Photonic Devices Based on Electro-Optic Modulation Technologies. Journal of the National Institute of Information and Communications Technologies, 53(3), 25-32, 2006.

[7] POBERAJ, G., HU, H., SOHLER, W., GUNTER, P.: Lithium Niobate on Insulator (LNOI) for Micro-Photonic Devices. Laser Photonics Reviews, 6(4), 488-503, 2012.

[8] WEIGEL, P.O., et al.: Lightwave Circuits in Lithium Niobatethrough Hybrid Waveguides with Silicon Photonics.Scientific Reports, 6, 22301, 2016.

[9] GORBACH, A.V., DING, W.: Microfiber-Lithium Niobateon Insulator Hybrid Waveguides for Efficient and Reconfigurable Second-Order Optical Nonlinearity on a Chip. Photonics, 2, 946-956, 2015.

[10] CHEN, L., NAGY, J., REANO, R.M.: Patterned Ion-Sliced Lithium Niobate for Hybrid Photonic Integration on Silicon. Optical Materials Express, 6(7), 2460-2467, 2016.

[11] CHEN, L., CHEN, J., NAGY, J., REANO, R. M.: Highly Linear Ring Modulator from Hybrid Silicon and Lithium Niobate. Optics Express, 23(10), 13255-13264, 2015.

[12] BAZZAN, M., SADA, C.: Optical Waveguides in Lithium Niobate: Recent Developments and Applications. Applied Physics Reviews, 2, 040603-1-040603-25, 2015.

[13] MAILIS, S., et al.: Direct Ultraviolet Writing of Channel Waveguides in Congruent Lithium Niobate Single Crystals. Optics Letters, 28(16), 1433-1435, 2003. 
[14] MIZEIKIS, V., et al.: Direct Laser Writing: Versatile Tool for Microfabrication of Lithium Niobate. Journal of Laser Micro/ Nanoengineering, 7(3), 345-350, 2012.

[15] PAIPULAS, D., BUIVIDAS, R., JUODKAZIS, S., MIZEIKIS, V.: Local Photorefractive Modification in Lithium Niobate Using Ultrafast Direct Laser Write Technique. Journal of Laser Micro/Nanoengineering, 11(2), 246-252, 2016.

[16] VITTADELLO, L., et al.: Photorefractive Direct Laser Writing, Journal of Physics D: Applied Physics, 49, 125103, 2016.

[17] YEH, P.: Introduction to Photorefractive Nonlinear Optics, Chapter 3. John Wiley \& Sons, Inc., New York, p. 82-117, 1993.

[18] TARJANYI, N.: Real-Time Imaging of Grating Formation in $\mathrm{LiNbO}_{3}$ :Fe Using Mach-Zehnder Interferometer. Optical Engineering, 49(8), 085602, 2010.

[19] KOGELNIK, H.: Coupled Wave Theory for Thick Hologram Grating. Bell System Technical Journal, 48, 2909-2947, 1969.

[20] TARJANYI, N., KACIK, D., TARJANYIOVA, G.: Light-Induced Microstructures in LiNbO ${ }_{3}$ Fe Crystal in Photonic Crystal Fibers. Proceeding of SPIE 6588, 658812, 2007.

[21] TARJANYI, N.: Specially Shaped Negative Lens Produced in Lithium Niobate Crystal. Optical Engineering, 53(5), $057104,2014$.

[22] ZILINSKA UNIVERZITA V ZILINE: System of Dioptric Elements from Photorefractive Material (in Slovak). Inventors: TUREK, I., TARJANYI, N., DUBRAVKA, M., G02B 17/00.UV 4988, Industrial property office of the Slovak Republic, 2007.

[23] TARJANYI, N., TUREK, I.: Influence of Surroundings on Photorefractive Effect in Lithium Niobate Crystals. Physica B, 407, 4347-4353, 2012.

[24] TARJANYI, N., KACIK, D.: Fabrication and Evaluation of Photorefractive Vaweguide in $\mathrm{LiNbO}_{3}$ :Fe, in Integrated Photonics: Materials, Devices and Applications. Proceeding of SPIE 8069, 80690B, 2011. 